Providing a nurse led referral pathway for an SRH in-reach clinic is acceptable, feasible and beneficial for PICU patients.

\section{Comparison of self-esteem and depression among fertile and infertile women}

\author{
Akani Ruth $^{1 \star}$ and Akinyemi Akintayo ${ }^{2}$ \\ ${ }^{1}$ Lincolnshire partnership NHS foundation trust and ${ }^{2}$ Piedmont \\ Athens regional medical center \\ ${ }^{*}$ Corresponding author.
}

doi: 10.1192/bjo.2021.176

Aims. This study aimed to explore the effect of infertility on selfesteem and depression, and to identify the sociodemographic and infertility characteristics associated with self-esteem and depression among infertile women in Ekiti State, Nigeria.

Method. Self-esteem and depression were assessed in 100 infertile women and 100 women seeking family planning (controls) using the Rosenberg Self-Esteem Scale (RSES) and Patient Health Questionnaire (PHQ-9), respectively. PHQ-9 score $\geq 10$ was defined as major depressive disorder (MDD). Continuous variables were presented as mean \pm standard deviation and categorical variables as frequency (percentage). Cases and controls were compared using Student's t test. $\chi 2$ or Fisher's exact (when cell size $<5)$ tests were performed to compare proportions. Simple and multiple linear regression analyses were used to examine the association between the sociodemographic, infertility characteristics and RSES or PHQ-9 scores among infertile women

Result. Infertile women had significantly lower RSES score (19.4 \pm 4.5 vs. $20.7 \pm 4.4, \mathrm{p}=0.038)$ and higher PHQ-9 score $(5.1 \pm 4.1$ vs $3.8 \pm 3.5, \mathrm{p}=0.023)$ compared to controls. Seventeen infertile women (17\%) and 8 women in the control group (8\%) had MDD (PHQ-9 score $\geq 10$ ) and were referred for further evaluation. Among infertile women, marital status, being remarried, duration of infertility, and RSES score were associated with PHQ-9 score on simple linear regression. Similar association was not seen in the controls. On multiple linear regression analysis, RSES score had a negative association with PHQ-9 score $(\beta=-0.32, p<$ 0.001 ) among infertile women. Older age [OR (95\% CI):1.13 $(1.01-1.25) ; \mathrm{p}=0.030], \leq 6$ years formal education [OR $(95 \%$ CI): $4.76(1.13-20.00) ; \mathrm{p}=0.033$ ], being remarried [OR $(95 \%$ CI): $10.87(1.86-63.64) ; \mathrm{p}=0.008]$, longer duration of infertility [OR (95\% CI): $1.11(1.01-1.22) ; \mathrm{p}=0.040]$ and RSES score [OR (95\% CI): $0.79(0.67-0.92) ; \mathrm{p}=0.003]$ were significantly associated with MDD. On multiple logistic regression analysis, only the association between RSES score and MDD remained statistically significant $(\mathrm{p}=0.004)$

Conclusion. Infertile women have lower self-esteem and higher depression scores in comparison to women seeking family planning. Mental health screening and management should be an integral part of care administered to infertile women

\section{A natural language processing approach to modelling treatment alliance in psychotherapy transcripts}

Jihan Ryu*, Stephen Heisig, Caroline McLaughlin, Rebeccah Bortz, Michael Katz and Xiaosi Gu

Icahn School of Medicine at Mount Sinai

${ }^{\star}$ Corresponding author.

doi: 10.1192/bjo.2021.177

Aims. Patient-therapist alliance is a critical factor in psychotherapy treatment outcomes. This pilot will identify language concepts in psychotherapy transcripts correlating with the valence of treatment alliance using natural language processing tools. Specifically, high-order linguistic features will be extracted through exploratory analysis of texts and interpreted for their power to discriminate alliance rated by patients.

Method. Adult patients and therapists in outpatient clinic at various stages of relationship building and treatment goals consented to participate in the cross-sectional study approved by the Institutional Board Review. Psychotherapy sessions were recorded using wireless microphones and transcribed by two research assistants. After the recording, each patient completed Working Alliance Inventory- Short Form, to generate clinical scores of alliance. We used the Linguistic Inquiry Word Count (LIWC) tool to map words to psycholinguistic categories, and generated novel linguistic parameters describing the individual language for each speaker role. Canonicalcorrelational analysis and descriptive statistics were used to analyze the two datasets.

Result. Patients $(\mathrm{N}=12,83 \%$ female, mean age $=40)$ were primarily diagnosed with personality disorders (67\%) working on real-life interpersonal issues (median treatment duration 18.5 weeks, 50\% psychodynamic, $32 \%$ cognitive-behavioral, $16 \%$ supportive modality). In this heterogenous sample, patients who used the "achieve" (e.g. trying, better, success, failure) and "swear" psycholinguistic categories of words rated the treatment alliance lower $(\mathrm{r}=-0.70$, $\mathrm{p}=0.01 ; \mathrm{r}=-0.65, \mathrm{p}=0.02$ ). Patients rated alliance lower with therapists, who used more "I" pronoun $(\mathrm{r}=-0.58, \mathrm{p}<0.05)$ and higher with therapists using more "risk" (difficult, safe, crisis) and "power" (important, strong, inferior, passive) categories $(\mathrm{r}=$ $0.66, \mathrm{p}=0.02, \mathrm{r}=0.58, \mathrm{p}<0.05$ ), which commonly appeared in psychoeducation and conceptual framing of problems. Interestingly, there was no correlation with "affiliation" category $(p=0.9)$. Linear regression modeling from "achieve," "swear" variables and "I," "risk" variables with duration of treatment as covariate predicted the patient's rating of alliance (Adjusted $\mathrm{R} 2=0.66, \mathrm{p}=0.03$ ). Conclusion. Our data collection and sub-sample analysis are ongoing. Preliminary results are showing speaker-specific language patterns in cognitive-emotional domain, e.g. self-expressivity, and in clinician's therapy style, covarying with the patient's perceived closeness in the heterogenous treatment dyads. Novel application of natural language processing to characterize alliance using the data-driven approach is an unbiased method that can provide feedback to clinicians and patients. This characterization can also potentially provide insights into the mechanisms underlying the therapeutic process and help develop psycholinguistic markers for this critical clinical phenomena.

Remotely connected: patient and clinician video care experiences in secondary mental health services during COVID-19, including future preferences

Lamiya Samad ${ }^{1 \star}$, Bonnie Teague ${ }^{2}$, Karen Moreira ${ }^{3}$, Sophie Bagge ${ }^{4}$, Khalifa Elzubeir ${ }^{5}$ and Jonathan Wilson ${ }^{6}$

${ }^{1}$ Norfolk and Suffolk NHS Foundation Trust, Medical Adviser represent UCL Great Ormond Street Institute of Child Health, to British Paediatric Surveillance Unit, Royal College of Paediatrics and Child Health; ${ }^{2}$ Norfolk and Suffolk NHS Foundation Trust, University of East Anglia; ${ }^{3}$ Norfolk and Suffolk NHS Foundation Trust; Emma Marriott, Norfolk and Suffolk NHS Foundation Trust; ${ }^{4}$ Norfolk and Suffolk NHS Foundation Trust; ${ }^{5}$ Norfolk and Suffolk NHS Foundation Trust and ${ }^{6}$ Norfolk and Suffolk NHS Foundation Trust, University of East Anglia ${ }^{\star}$ Corresponding author.

doi: 10.1192/bjo.2021.178 
Aims. Video-delivered care is a rapidly emerging area with potential to transform assessment and treatment strategies. The coronavirus (COVID-19) pandemic has accelerated these changes. Limited evidence exists for experiences of video care in secondary mental health services. We aimed to assess the acceptability of video care in mental health clinical practice during COVID-19.

Method. Structured questionnaires were developed with the help of patients and clinicians. The patient experience questionnaire was built into video sessions and completed online, using the Attend Anywhere (AA) platform from July 2020 to March 2021. A Trust-wide clinician views and experiences survey was conducted from July 2020 to October 2020. Descriptive analysis was performed using SPSS (version 27.0).

Result. Of 1,296 patients who completed the online feedback, the majority provided positive feedback for all aspects of video care. Most patients felt their needs were met (90\%) and were supported (93\%) during the video call. Positive experiences were informed by clinicians' communication skills. For future appointments, just over half $(51.7 \%)$ of patients preferred using video calls, followed by face-to-face (33\%). Future video preference was informed by reasons reducing social anxiety and practical aspects such as child/carer needs, physical disability and travel.

Of 252 clinicians completing the survey, 161 (64.7\%) had used video for remote care delivery. Clinicians also provided positive feedback, with Microsoft-teams as the preferred platform. Most clinicians felt the therapeutic relationship (76.4\%) and privacy $(78.7 \%)$ were maintained using video. While $73 \%$ felt there were no safeguarding issues that impacted adversely, $30 \%$ felt that care quality was affected, and (69.9\%) reported limited visual cues for video calls. Most clinicians (73\%) felt confident about clinical decision-making remotely, though there were areas where clinicians felt less confident, such as assessing patients' appearance and behaviour. Additionally, compared with face-to-face, video consultations seemed to be effective for social anxiety, but less so for Autism spectrum disorders, and with no perceived difference for depression or self harm. For future, more clinicians preferred face-to-face $(40.1 \%)$ than video care $(36.1 \%)$.

Conclusion. Mental health care delivered remotely via video is experienced positively by patients and clinicians alike. However, clinicians felt that quality of care is impacted, and additional remote clinical skills training may be beneficial. Going forward, there is acceptability for the use of video care in routine mental health practice for certain mental health presentations.

Sleep and the risk of cognitive impairment and dementia: further insights from the Caerphilly Prospective Study

James Selwood $^{1 \star}$, Elizabeth Coulthard ${ }^{1}$, Antony Bayer ${ }^{2}$, Mark Fish $^{3}$, John Gallacher ${ }^{4}$ and Yoav Ben-Shlomo ${ }^{1}$

${ }^{1}$ University of Bristol; ${ }^{2}$ University of Cardiff; ${ }^{3}$ Taunton and Somerset NHS Foundation Trust and ${ }^{4}$ University of Oxford

${ }^{*}$ Corresponding author.

doi: 10.1192/bjo.2021.179

Aims. Sleep disorders are highly prevalent with growing evidence that sleep problems may predict cognitive decline and dementia. A previous analysis of the Caerphilly Prospective Study (CaPS), a cohort of middle-aged men, found that daytime sleepiness predicted vascular dementia and cognitive impairment. We have re-examined this hypothesis with additional events based on further follow-up. The study aimed to examine the role of different sleep problems in predicting cognitive impairment not dementia (CIND) and dementia. Our hypothesis was that sleep problems in mid-life would predict CIND and dementia in later life.

Method. CaPS is a population cohort of men born between 1920 and 1935 and resident in Caerphilly in South Wales, first seen between 1979-1983. Cognitive tests and a sleep questionnaire were introduced at Phase III (men aged 55-69 years). The questionnaire asked about daytime dysfunction, hypnotic use, insomnia, napping, nocturnal limb movements, sleep apnoea, sleep duration, sleep latency and snoring.

At Phase V (men aged 68-82 years), poor performance on the Cambridge Cognition Exam (CAMCOG) was used to select men for detailed clinical assessment. Subjects were classified as having normal cognition, CIND or dementia. Cognitive disorders were sub-classified as vascular or non-vascular. At Phase VII (men aged 78-92 years), new cases were identified and survivors with existing diagnoses were reassessed.

We initially conducted separate logistic regressions for vascular and non-vascular cognitive outcomes with the individual sleep measures, but where there was no evidence of heterogeneity, we combined these outcomes to enhance power. We also ran ordered logistic regression models to test for association of our sleep measures with CIND and dementia from any cause, with no cognitive problems as the reference group. All models were adjusted for potential confounders such as age and lifestyle variables.

Result. There were 256 cases of CIND, 155 dementia and 118 vascular cognitive disorders. 889 had normal cognition. Nocturnal limb movements strongly predicted vascular cognitive disorders (OR 2.59, 95\% CI 1.34-4.98, $\mathrm{p}=0.004$ ). Poor sleep duration, defined as less than 6 or more than 8 hours, predicted all-cause CIND and all-cause dementia (OR 1.62, 95\% CI 1.01-2.61, $\mathrm{p}=0.045)$. The other sleep measures showed weak associations consistent with chance.

Conclusion. We have provided further evidence that sleep problems predict cognitive decline, justifying the growing interest in sleep as a potentially modifiable risk factor for dementia. Future evidence is required from intervention studies that attempt to improve sleep parameters.

\section{Mechanisms of transcranial magnetic stimulation in the treatment of anorexia nervosa}

\author{
Lydia Shackshaft \\ King's College London
}

\section{doi: 10.1192/bjo.2021.180}

Aims. Severe and Enduring Anorexia Nervosa (SE-AN) is a challenging condition to treat, with limited therapeutic options, high morbidity, and the highest mortality rates of any psychiatric illness. Repetitive Transcranial Magnetic Stimulation (rTMS) is an emerging treatment option, as evidence demonstrates promising efficacy in improving mood and reducing core Anorexia Nervosa symptoms, as well as safety and tolerability to patients. We aimed to investigate the neurophysiological mechanisms of rTMS use in SE-AN patients by assessing changes in resting state functional connectivity, in the first functional neuroimaging analysis investigating rTMS effects in Anorexia Nervosa patients. Method. 26 females with a current diagnosis of SE-AN received 20 sessions of sham or real high frequency rTMS (10 hertz) to the left dorsolateral prefrontal cortex in a randomised doubleblind trial. Resting-state functional magnetic resonance imaging was performed before and after rTMS. Neural correlates of 